Georges DE LEENER

Professeur à l'Université de Bruxelles

\title{
LES CHEMINS DE FER
}

\section{EN BELGIQUE}

Extruit de la Revue Économique Internationale (novembre 1926).

\section{BRUXELLES}

Gozmaere, Imprimeur du RoI, ÉdTteur,

Rue de la Limite, $2 I$ 


\section{LES CHEMINS DE FER EN BELGIQUE.}

SOMMAIRE : La crise du Trésor et la mobilisation d'un élément de l'actif de l'Etat pour y parer. - Constitution de la Société Nationale des Chemins de fer belges : caractères essentiels de la Société. - L'exploitation du chemin de fer par l'Etat avant la guerre et depuis l' armistice. - Importance du réseau et du trafic. - Les nouvelles conditions d'exploitation. Conclusions.

SUMMARY : Treasury crisis and the mobilization of an element of the State assets to meet it. - Founding of the National Belgian Railroad Company: essential character of the company. - Exploitation of railroad by the State before the war and since the armistice. - Importance of the lines and the trafic. - New conditions of exploitation. - Conclusions.

A constitution, le 7 août 1926 , de la Société nationale des Chemins de fer belges, a consacré le transfert, à une société nouvelle, de l'exploitation des chemins de fer de l'État belge. Cette cession a été motivée, avant tout, par des préoccupations financières.

Les finances de l'État traversaient, depuis plusieurs mois, la crise la plus grave qu'elles eussent subie depuis la guerre. Après un échec retentissant de sa stabilisation sur la base de la livre sterling à 107, le franc belge s'était déprécié rapidement au point que la livre sterling avait été portée en quelques bonds rapides jusqu'au cours de 
240 francs. On pouvait craindre que, la dépréciation continuant à s'aggraver, le franc belge fût emporté dans une débâcle irrésistible. Les conséquences les plus funestes auraient bientôt suivi. Elles se seraient manifestées surtout par le déséquilibre complet du budget de l'État, pour lequel la charge de la dette extérieure serait devenue écrasante.

Le salut financier de la Belgique fut la tâche à laquelle se consacra d'emblée un Gouvernement d'union nationale, constitué le 20 mai r926. Il obtint immédiatement du Parlement les pouvoirs nécessaires pour la création, sous le nom de Fonds d'amortissement de la Dette publique, d'un organisme chargé, notamment, de réduire la dette flottante. Celle-ci avait été le principal obstacle à la réussite de la stabilisation monétaire entreprise en 1925 et elle exposait l'État à l'évenfualité du remboursement dans les plus prochains mois, d'une somme totale de 5 milliards 800 millions, sans compter la dette flottante extérieure.

Quand une entreprise souffre d'un défaut de trésorerie qui la menace de déconfiture, ses dirigeants s'ingénient à utiliser tous les éléments de son actif pour lui procurer les disponibilités nécéssaires. Ce procédé n'a rien que de très normal. Si son fonds est solide, l'entreprise opérera un rétablissement, après quoi elle pourra connaître la plus grande prospérité. Tel est le principe qui devait inspirer M. le Ministre d'État Francqui lorsqu'il élabora le projet réalisé sous la forme de laSociété nationale des chemins de fer belges.

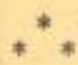

La Société nationale des chemins de fer belges constitue une nouveauté dans le mode d'exploitation des voies ferrées. Sans cesser d'appartenir à l'État, les chemins de fer sont exploités par une entreprise autonome. Celle-ci assure 
l'industrialisation en même temps que l'autonomie. L'une et l'autre étaient depuis longtemps les objectifs d'efforts tendant à soustraire l'exploitation des chemins de fer de l'État belge à l'action débilitante de l'État.

La nouvelle société est d'abord - et son nom même l'atteste - une société au sens strict du mot,avec un capital qui est le bien propre de ses actionnaires. Son capital est fixé à 11 milliards de francs, représentés par Io millions d'actions ordinaires de 100 francs chacune et par 20 millions d'actions privilégiées de 500 francs chacune. Les unes et les autres ont été remises à l'État en rémunération de sa cession de l'exploitation de ses chemins de fer; mais tandis que les actions ordinaires ne peuvent être aliénées et seront conservées en portefeuille par l'État, les actions privilégiées ont été cédées par ce dernier au Fonds d'amortissement de la Dette publique. Celui-ci en dispose aux fins mêmes pour lesquelles il a été constitué. Dans ce but il en a émis par voie de souscription publique et il en a échangé contre des Bons du Trésor. Ces titres jouissent de la garantie d'un intérêt qui leur sera payé par l'État. Le taux en sera fixé à chaque émission d'après la situation du marché de l'argent. Ils interviennent, en outre, dans les bénéfices d'exploitation de la Société nationale à partager par moitié avec les actions ordinaires. D'autre part, l'État conserve à sa charge le service de la partie de la Dette publique contractée pour lă construction des chemins de fer.

La direction de la Société nationale des chemins de fer belges est confiée à un conseil d'administration composé de vingt et un membres, dont dix-huit nommés par le Roi et trois par le personnel. Les membres nommés par le Roi sont choisis partie à raison de leur compétence particulière, partie sur la proposition du Conseil d'administration du Fonds d'amortissement de la Dette publique, partie parmi 
les candidats présentés par les Conseils supérieurs de l'agriculture, de l'industrie et du commerce, et du travail.

Les dispositions de la loi qui a institué la Société nationale en ont fait une entreprise jouissant d'une réelle autonomie et engageant la responsabilité des administrateurs, à l'égal des sociétés anonymes. On remarquera tout particulièrement que le Conseil d'administration ne dépend aucunement du Parlement, puisque, à trois membres près, il est entièrement nommé par le Roi, et encore ces trois membres sont-ils désignés non par les Chambres, mais par le personnel. Le Conseil d'administration n'est tenu à aucune formalité de présentation de budget. C'est tout au plus s'il est tenu de communiquer aux Chambres le bilan et le compte de profits et pertes annuels, pour simple information. Ils ne sont soumis à aucun vote de ces assemblées.

Pareille autonomie permet d'attendre de la Société nationale des chemins de fer belges des résultats d'exploitation très satisfaisants. L'ancienne exploitation par l'État subissait les pires inconvénients par le fait de l'ingérence gouvernementale et de l'intrusion parlementaire. L'État n'achetait pas toujours au mieux. Des nécessités d'opportunisme obligeaient les ministres responsables à acheter à l'intérieur ce qu'ils auraient pu acquérir à l'étranger à moindre prix. L'État était enclin, sous des pressions politiques, à accorder des tarifs de faveur, des augmentations de traitement qui ne se justifiaient pas toujours, et des avantages qui profitaient à l'arrondissement de tel ou tel membre de la majorité du Gouvernement. A ce point de vue l'État se trouvait évidemment dans de mauvaises conditions. pour gérer son réseau de chemins de fer. L'administration des Chemins de fer de l'État belge n'avait pas su se garder non plus de dépenses somptuaires incompatibles avec une 
bonne gestion. On a souvent cité l'exemple de la gare centrale d'Anvers qui avait coûté 30 millions de francs d'avantguerre sans qu'elle satisfasse ni aux exigences du service ni aux conditions de confort d'une gare moderne. On aurait pu l'établir un kilomètre plus loin. On aurait disposé ainsi de plus de place et une construction plus simple eut suffit. Il en aucait coûté à l'État 25 millions de moins.

Aussi le coût kilométrique des lignes de l'État belge avaitil atteint en I9I3 une moyenne de 655,325 francs, dépassant d'environ 270,000 francs le coût kilométrique du réseau de l'État prussien et de plus de 100,000 francs celui du réseau de la Compagnie du Nord. Et ce qui rendait cette constatation plus inquiétante, ajoutait M. Vanderrydt, à qui nous empruntons ces considérations, c'est que l'augmentation annuelle du capital engagé par kilomètre suivait sur notre réseau une progression beaucoup plus rapide que sur les autres. De rgoo à rgro l'augmentation du coût du kilomètre avait été de 26.6 p. c. à l'État belge, tandis qu'il n'était que de 13.8 p. c. à l'État prussien, de 14.5 p. c. à l'État saxon, de Ir.2 p. c. à la Compagnie du Nord et de Ir.9 p. c. à la Compagnie de l'Est, sans que la construction de lignes nouvelles eut plus d'importance dans notre pays que dans les autres (I).

Cette exagération des charges financières des chemins de fer de l'État belge était en partie la conséquence de la lenteur déplorable avec laquelle il construisait les lignes nouvelles et des intérêts intercalaires très élevés qui s'ajoutaient aux capitaux directement immobilisés dans la construction. D'autre part,les chemins de fer de l'État belge employaient, en r912, 0.98 locomotive par kilomètre exploité, alors que le nombre correspondant était de 0.53 à l'État prussien et de 0.57 à la Compagnie du Nord.

(1) Cl. Instituts Solvay. Institut de Sociologie. L'autonomie des chemins de fer de TEtat belge. Bruxelles, J. Lebègue et Cin, 1919, pp. 3 et ss. 
Cette différence considérable n'était pas justifiée par les besoins de l'exploitation. Elle résultait de ce que l'administration belge avait acheté beaucoup de locomotives de puissance insuffisante et, qu'au lieu de démolir les locomotives surannées et pour ainsi dire inutilisables, elle les avait maintenues dans son effectif et avait acheté en extension des machines nouvelles, afin de reporter sur le compte capital des dépenses qu'elle aurait dô imputer sur le compte exploitation.

Ces causes qui avaient fait monter à un taux excessif le coût au kilonmètre du réseau des chemins de fer de l'État belge, $\mathrm{y}$ compris tout son matériel, étaient sous l'influence de la confusion du budget des chemins de fer avec celui de l'État.Elles se ressentaient aussi des effets de l'action directe que le Gouvernement n'avait cessé d'exercer sur la gestion de nos voies ferrées. Aujourd'hui, on peut dire, si l'on se place au point de vue de la Société nationale des chemins de fer belges, qu'elles appartiennent au passé; car, grâce à sa charte fondamentale, la nouvelle société n'a rien assumé des charges financières qui grèvent le réseau dont l'exploitation lui a été cédée. Ces dettes sont restées entièrement à charge de l'État. Par conséquent, elle ne pâtira en rien des défauts les plus essentiels de la constitution du réseau des chemins de fer de l'État belge. Abstraction faite de l'exagération du capital immobilisé, on peut remarquer que leur exploitation se présentait déjà sous l'ancienne administration dans des conditions favorables, dont le coefficient d'exploitation nous donnera la mesure pour les dix dernières années d'avant-guerre :

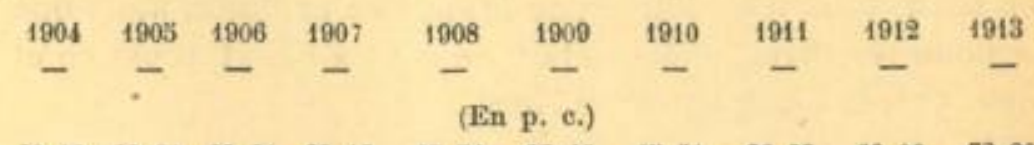

$\begin{array}{llllllllll}60.03 & 62.60 & 63.81 & 69.18 & 67.74 & 67.46 & 65.61 & 66.89 & 69.00 & 72.80\end{array}$ 
Ces coefficients d'exploitation révèlent une situation favorable, surtout eu égard à l'extrême modicité des tarifs qui étaient perçus avant la guerre sur les chemins de fer de l'État belge.

Les tarifs de $\mathrm{I}^{\mathrm{ro}}$ classe, de $3^{\circ}$ classe et pour ouvriers étaient (en centimes-or par kilomètre-voyageur) $[\mathrm{r}]$ :

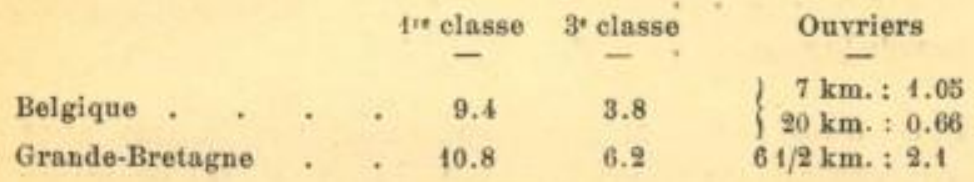

A titre documentaire, nous ajouterons que depuis l'armistice le coefficient d'exploitation a varié comme suit :

$\begin{array}{ccccccc}1919 & 1920 & 1921 & 1922 & 1923 & 1924 & 1925 \\ - & - & - & -56 & - & - & - \\ 120.20 & 98.84 & 86.55 & 76.75 & 93.90 & 90.25 & 93.33\end{array}$

L'augmentation du coefficient d'exploitation depuis l'armistice est le résultat avant tout du manque de réajustement des tarifs à la dépréciation de l'argent. Elle se constate, d'ailleurs, aussi sur les réseaux étrangers, tels que celui de la Compagnie du Nord, où le coefficient d'exploitation est passé de 61.32 p. c. en I 913 à 89.6 p. c. en I923, pour retomber à 79.5 en 1924 et $79 . \mathrm{I}$ en 1925 . Le coefficient réduit de 76.75 en 1922 s'explique par la grande prospérité qui se manifesta cette année en Belgique. Les derniers coefficients connus révèlent une situation très favorable, à savoir 82 p. c. en juillet 1926 contre 88.7 p. c. en juillet 1925 . Le chiffre de juillet 1926 est d'autant plus significatif qu'il a été constaté avant le dernier relèvement des tarifs d'octobre 1926 .

(1) Rapport de M. le général H.-O. Mance et de M. Jules Jadot au sujet des recettes nettes que les chemins de fer dé l'État belge, exploités commereialement, pourraient fournir en vue de financer le remboursement de la dette flottante belge. P. $\$$. 
Pour ce qui concerne particulièrement la Belgique, les mêmes tarifs ressortaient en mars 1926 , en centimes-or, à un taux sensiblement moins élevé que celui de Igr $_{3}$, exception faite pour les transports d'ouvriers à petite distance.

\begin{tabular}{|c|c|c|c|}
\hline & $1^{\text {tre elasse }}$ & 20 classe & Ouvriers \\
\hline . . . . & 7.6 & 3.0 & $\left\{\begin{array}{r}7 \mathrm{~km}: 1.08 \\
20 \mathrm{~km}: 0.56\end{array}\right.$ \\
\hline Grande-Bretagne. & 16.9 & 9,3 & $61 / 2 \mathrm{~km}: 4.7$ \\
\hline
\end{tabular}

On voit aussi par la comparaison des tarifs belges avec les tarifs anglais, combien, après l'armistice, les premiers étaient restés extraordinairement modiques jusqu'au courant de 1926.

En dépit de l'exagération des charges financières et des défauts de la confusion de l'exploitation des transports avec les administrations de l'État, les chemins de fer de l'État belge avaient pu néanmoins produire des résultats avantageux en procurant à leurs usagers des transports à bon marché. C'était l'effet des conditions éminemment favorables que la Belgique offre au développement du trafic des voies ferrées.

La première de ces conditions est liée au rôle de la Belgique comme pays de transit. Les créateurs de nos voies ferrées avaient eu, dès r 833 , une véritable divination des avantages que notre pays était en état d'attendre de sa situation géographique.

On connaît les projets inspirés à Napoléon par le désir de réunir l'Escaut à la Meuse et la Meuse au Rhin. Tous les gouvernements auxquels la Belgique fut tour à tour soumise avaient toujours caressé les mêmes espoirs. Napoléon voulut passerer à l'exécution en faisant creuser des canaux; mais les événements de $\mathrm{I} \mathrm{I}_{4}$ ayant arrêté les tra- 
vaux commencés en 1808 , il ne resta de ces projets que le canal de Reckheim à Bocholt, achevé par le gouvernement des Pays-Bas.

Ce souci n'eût pas été de tous les temps si la Belgique n'avait pas paru prédestinée à attirer par ses conditions naturelles un transit considérable. Celui-ci devait résulter de la situation géographique du pays entre les régions les plus peuplées et les plus industrielles de l'Europe occidentale, d'une part, et l'estuaire de l'Escaut, largement ouvert à la grande navigation maritime, d'autre part. Le même transit devait être affecté en outre par le voisinage de l'Angleterre qui exerçait depuis longtemps une attirance irrésistible sur les courants commerciaux de l'Europe continentale.

C'est donc la situation géographique qui explique l'abondance du transit qui traverse la Belgique. L'importance même de ce transit ressort des statistiques officielles du commerce extérieur. Pendant l'année 1924, 13.420.254 tonnes de marchandises les plus diverses ont été transportées à travers la Belgique et près de la moitié ont emprunté la voie ferrée. Ce tonnage joue un très grand rôle dans l'exploitation de nos voies ferrées. Il peut être l'élément d'un sérieux rendement pour la nouvelle Société nationale des chemins de fer belges. S'il est vrai que, d'une part, les voies ferrées étrangères et les voies navigables disputent à nos chemins de fer au moins une partie de ce transit, celui-ci offre cependant, d'autre part, l'avantage de transports sur de longues distances dans des conditions qui les rendent les plus rémunérateurs.

Le transit doit aussi son importance spéciale pour la Belgique à l'insuffisance des seuls transports intérieurs pour fournir aux voies ferrées un trafic suffisant. Cette insuffisance est le fait du grand développement du réseau ferré belge par rapport à l'étendue du pays. A ce grand déve- 
loppement correspond un investissement de capitaux qui ne pourraient être convenablement rémunérés sans l'appoint du transit.

A côté de l'action du transit sur l'exploitation des voies ferrées en Belgique, la forte densité de la population de notre pays est un autre facteur non moins considérable. A cette grande densité de population -254 habitants par kilomètre carré - correspond une extrême multiplicité de villes et de villages dispersés sur toute l'étendue du pays. Le besoin de communications rapides et à bon marché chez leurs habitants a nécessité l'établissement d'un très grand nombre de stations et de haltes de chemins de fer, encore que le développement du réseau des chemins de fer vicinaux eút permis de satisfaire en partie à ce besoin. Les habitants se déplaçant d'autant plus facilement d'un endroit à l'autre que les distances sont plus petites, le grand nombre de leurs déplacements a entraîné la mise en service de nombreux trains de voyageurs qui sont tous très achalandés.

Pendant l'année 1913 , le nombre de voyageurs transportés sur les chemins de fer de l'État et sur ceux des compagnies a atteint le chiffre de 224.250.000. Abstraction faite des voyageurs étrangers, ce chiffre correspond à une moyenne de près de 30 voyages par habitant et par an, sur la base de la population au 31 décembre de la même année. Sur les seuls chemins de fer de l'État, le nombre des voyageurs transportés en 1925, s'est élevé à 222746.000 , avec une distance moyenne de transport d'environ 27 kilomètres.

La grande densité de la population distribuée sur toute l'étendue du pays se prête à un drainage de la main-d'œuvre requise pour les besoins de l'industrie qui est plus ou moins concentrée dans les villes et dans des régions industrielles. Ce drainage fournit aux transports par chemin de fer un nombre considérable de voyageurs journaliers ou hebdoma- 
daires. On l'a évalué depuis l'armistice à plus de 300,000 .

La densité de la population mérite de retenir encore notre attention pour un autre effet intéressant au point de vue du rendement de l'exploitation des voies ferrées. Il est admis, en cette matière, que toute réduction de tarif a d'autant plus d'effet sur l'augmentation de trafic que le réseau est à la fois plus étendu et plus dense. Dans le cas de notre pays, la condition de grande étendue du réseau n'est pas remplie; par contre, notre réseau des chemins de fer satisfait pleinement à la condition de grande densité des lignes. Plus nombreuses et resserrées sont celles-ci, et plus un grand nombre de personnes sont en situation de profiter de réductions éventuelles de tarifs, soit pour se déplacer, soit pour faire par chemins de fer des expéditions de marchandises, ou pour en recevoir. Aussi les augmentations consécutives de trafic peuvent-elles compenser à l'avantage de l'exploitant les réductions de tarifs qu'il consentirait.

Une population de $7,750,000$ habitants, vivant sur un étroit territoire de 30,440 kilomètres carrés, est contrainte à des échanges intensifs avec l'étranger, sous peine de périr d'inanition. Pour se nourrir, elle a besoin d'importations de gros tonnages de produits alimentaires. Pour travailler, elle acquiert au dehors de grandes quantités de matières premières. Pour gagner de quoi acquérir ses aliments, elle exporte en abondance les produits de son industrie. Située à proximité immédiate de la mer, et mieux encore du port d'Anvers, la Belgique est en état de recevoir des matières premières très pondéreuses, sans qu'elles soient grevées de frais de transport excessifs. Elle peut, pour la même raison, se livrer à des industries d'exportation dont les produits sont des marchandises lourdes. Produits alimentaires importés en grandes quantités, matières premières pondéreuses acquises au dehors, gros produits exportés à l'étranger, 
voila de quoi fournir aux exploitants des chemins de fer en Belgique un très grand tonnage de transport ! Cette triple constatation est d'ailleurs en rapport avec le fait du grand commerce extérieur de la Belgique. On sait qu'elle n'est dépassée à cet égard que par un petit nombre de très grands pays.

Outre le transit de 13 I/2 millions de tonnes, le commerce extérieur de la Belgique a compris, en 1924, 33 I/2 millions de tonnes à l'importation et plus de 20 millions de tonnes à l'exportation. Même en tenant compte des transports intérieurs par eau, ces seuls chiffres expliquent, pour la plus grande partie, le grand tonnage des marchandises transportées sur les chemins de fer de l'État pendant la même année. Il a atteint, en effet, 7 I. 752.275 tonnes. Ce gros tonnage est expliqué, en outre, par la nature de la production belge pour les besoins mêmes du pays. A cet égard, quelques chiffres de production méritent d'être cités. Nous commencerons par l'extraction de la houille. La production nette annuelle est d'environ 20 millions de tonnes, dont la presque totalité, soit environ 18 millions de tonnes restent dans le pays après défalcation d'environ 2 millions de tonnes livrées à l'exportation. Les statistiques de l'administration des Chemins de fer de l'Etat nous révèlent d'ailleurs que les combustibles transportés sur tout le réseau, à quelque destination que ce fut, ont atteint $21,182,000$ tonnes en 1924 , et $20,008,000$ tonnes en 1925 . Ces quantités comprennent en outre les charbons étrangers importés en Belgique.

D'autres gisements minéraux s'ajoutent aux houillères pour fournir un tonnage considérable aux chemins de fer. Nous voulons parler des produits des carrières dont les chemins de fer de l'État belge ont transporté 5.803 .000 tonnes en 1924 et $5 \cdot 537.000$ tonnes en I925. Ces transports sont encore grossis par la chaux, le platre et le ciment avec 
2.622,000 tonnes en $\mathrm{r} 924$ et $\mathbf{2 . 5 3 7 . 0 0 0}$ tonnes en $\mathrm{r} 925$ et

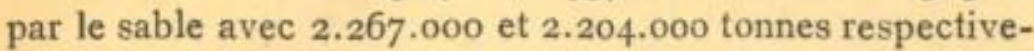
ment l'une et l'autre de ces années. Sans insister nous retenons encore pour le gros volume de transport qu'elles procurent aux chemins de fer, soit par leurs approvisionnements en matières premières, soit par leurs expéditions de produits fabriqués, quelques grandes industries nationales telles que l'industrie métallurgique, l'industrie des engrais, la fabrication des briques, les industries céramiques, les industries textiles, la verrerie et la glacerie.

L'entièreté de ces marchandises ne fait pas l'objet de transports par chemin de fer. Une partie en est détournée par la voie navigable; mais les transports intérieurs par eau ne font aux chemins de fer belges qu'une concurrence très atténuée. A ce point de vue une grande différence doit être soulignée entre la Belgique et des pays comme la Hollande ou l'Allemagne. Si des voies navigables existent en Belgique, elles laissent cependant aux chemins de fer la plus grosse part du trafic.

Tandis que le réseau des chemins de fer belges présentait, au 3 I décembre 1925 , un développement de 5.03I kilomètres en exploitation, sans compter 4.359 kilomètres de chemins de fer vicinaux, la longueur des voies navigables n'atteignait, à la même date, que r.990 kilomètres (I). Rien que cette différence suffirait déjà pour expliquer qu'une part prépondérante du trafic ḑoive nécessairement être opérée par la voie ferrée à.l'exclusion de la voie navigable. La petite distance des transports intérieurs en Belgique en rapport avec l'étroitesse du pays agit dans le même sens. Là même où les tarifs de chemins de fer sont

(1) D'après le Tableau annuel du eommerce avec les pays 6́trangers , publié par le Ministère des Finances de Belgique. Année 1924. Premier volume, p. 39. 
élevés, les voies navigables ne leur enlèveraient du trafic que pour les transports à grande distance. Cette division de trafic a donné lieu jadis à des études minutieuses en Allemagne. Elles ont démontré qu'en dessous d'une distance d'une cinquantaine de kilomètres, lors même que l'expéditeur et le destinataire se trouvaient situés directement au bord de la voie d'eau, le transport par chemin de fer restait plus avantageux que la navigation. C'était la conséquence des frais supplémentaires nécessaires si l'on a recours à cette dernière. Ils ne peuvent être compensés par le bon marché du déplacement de la marchandise par bateau que moyennant qu'elle soit transportée sur des distances sensiblement plus longues que la distance moyenne des transports intérieurs en Belgique. Cette distance moyenne a été en 1925 de 57 kilomètres pour les grosses marchandises ( $4^{\mathrm{m} \bullet}$ classe) transportées sur les chemins de fer de l'État belge.

Ces circonstances expliquent que les voies navigables laissent de beaucoup la plus grande partie du trafic aux voies ferrées. Sur ce point, plusieurs chiffres officiels sont péremptoires.

\section{AxNÉr 1924.}

Tonnes de marehandises Tonnes de marehandises transportées par

Nature du trafie ehemin de fer transportées par bateaux d'intérieur

$\begin{array}{lrc} & - & - \\ \text { Importations . * : } & 17,596,000 & 5,6 \times 2,000 \\ \text { Exportations : * : } & 10,627,000 & 3,165,000 \\ \text { Transit à l'entrée: } & 6,301,000 & 2,953,000 \\ \text { Transit à la sortie : } & 5,731,000 & 9,588,000\end{array}$

Les chiffres que nous venons de présenter au lecteur lui auront démontré que les chemins de fer conservent la préférence pour le transport des marchandises, même dans les relations extérieures, c'est-à-dire dans les conditions les 
plus favorables eu égard aux longues distances de transport. A plus forte raison, leur supériorité ressort-elle encore plus nettement dans les transports intérieurs. La statistique des transports de combustibles est particulièrement intéressante à ce point de vue. En 1912, 7.2 millions de tonnes de combustibles minéraux furent transportées sur les voies navigables (r). Défalcation faite de 5 millions de tonnes de houille étrangère importée, il reste environ 2.2 millions de tonnes de charbon belge transportées par bateau, soit à peine le dixième de la production. En d'autres termes, 90 p.c. de la production ont été expédiés par la voie ferrée. Cette proportion est d'autant plus significative - que le charbon est une marchandise qui par sa nature et ses conditions d'expédition en gros tonnage se prête mieux que toute autre aux transports par eau.

Sans doute, la concurrence de la voie navigable pourraitelle disputer plus sérieusement au chemin de fer une partie de son trafic si les canaux étaient réfectionnés et multipliés. De nombreux projets ont été dressés dans ce but. Il y a lieu de croire que les difficultés financières de l'État en différeront l'exécution pendant longtemps. La concurrence des voies navigables aux chemins de fer parait donc devoir rester très mitigée.

Les influences que nous venons de caractériser ont prédisposé les chemins de fer belges à une exploitation intensive sans pareille. Elles ont agi aussi d'emblée pour encourager l'établissement en Belgique, dès I834, de la première voie ferrée qui eut été construite sur le continent. L'objectif du gouvernement, qui en prit l'initiative dans les premiers

(1) Cf. A. Defrren. Le monvement des combustibles minéraus sur les voies navigables belges, Bruxelles. Imprimerie Nareisse, 1919. 
mois de 1831 , était précisément en rapport avec la situation de la Belgique comme pays de transit. Son principal souci était de conserver au pays, en dépit de sa toute récente séparation de la Hollande, tous les avantages de communications faciles avec l'Allemagne pour en détourner le trafic vers Anvers et même vers Ostende en le soustrayant à la concurrence des ports hollandais. Dans la suite, encore, le tracé de plusieurs lignes principales du réseau fut guidé par le même souci. L'exploitation des charbonnages particulièrement dans le Hainaut et dans la province de Liége détermina la multiplication des voies ferrées que nécessitait le développement de l'extraction charbonnière. L'expansion économique des régions frontières dans les pays voisins de la Belgique suscita la construction de nombreuses lignes de chemins de fer qui soudèrent aux leurs notre réseau de voies ferrées.

Les constructions successives de voies ferrées dans toutes les parties du pays, soit par l'État, soit par les compagnies et le rachat par l'État de la presque totalité des réseaux privés,portèrent à 4.725 kilomètres la longueur des voies exploitées par l'État en 1925. Le nombre de stations, haltes et points d'arrêt était de 1.263 au 31 décembre de la même année. Compte tenu d'environ 300 kilomètres de lignes exploitées par les compagnies ou en service commun avec l'État, la densité du réseau ferré de la Belgique revient à 162 mètres par kilomètre carré.

Outre les 222.746.00o voyageurs, dont le nombre a été indiqué plus haut, les chemins de fer de l'État ont transporté en 1925,7 r.124.537 tonnes de marchandises représentant 7 milliards et 66 millions de tonnes-kilomètres.

A ce trafic, qui est étroitement conditionné par les influences du milieu dans lequel sont exploités les chemins de fer belges, ont satisfait 38.144 .269 trains-kilomètres 
voyageurs et 31.658 .805 trains-kilomètres marchandises. Ces derniers chiffres font ressortir à 159 voyageurs par train l'utilisation moyenne des trains de voyageurs et à 223 tonnes de grosses marchandises par train l'utilisation moyenne des trains de marchandises. Le prix de revient moyen a été de ro.9 centimes par voyageur-kilomètre et de $\mathbf{2} 2.5$ centimes par tonne-kilomètre, tandis que les recettes moyennes ont été respectivement pour l'un et pour l'autre de 7.5 centimes et de 13.7 centimes. Ces chiffres accusent un déficit de 3.4 centimes par voyageur-kilomètre et un gain de 1.2 centime par tonne-kilomètre.

Avant la guerre déjà les transports de voyageurs étaient déficitaires, tandis que les transports de marchandises se soldaient presque en équilibre, à savoir, en $\mathrm{I}_{9} \mathrm{I} 3$ : Voyageurkilomètre : recette moyenne : $\mathbf{2} . \mathbf{3}$ centimes; prix de revient : 3 centimes. Tonne-kilomètre de grosse marchandise : recette moyenne: 3.6 centimes; prix de revient : 3.7 centimes.

Exception faite pour le service des voyageurs en I924 et 1925 , le trafic n'a cessé de croître.

\begin{tabular}{|c|c|c|c|c|}
\hline Année. & $\begin{array}{l}\text { Nombre de } \\
\text { voyageurs. }\end{array}$ & $\begin{array}{l}\text { Nombre de } \\
\text { voyageurs- } \\
\text { kilomètre. }\end{array}$ & $\begin{array}{c}\text { Nombre de } \\
\text { tonnes } \\
\text { de grosses } \\
\text { marehandises. }\end{array}$ & $\begin{array}{l}\text { Nombre de } \\
\text { tonnes-kilomètre } \\
\text { de grosses } \\
\text { marchandises. }\end{array}$ \\
\hline 1907. & $159,400,000$ & $3,715,500,000$ & $53,000,000$ & $4,878,100,000$ \\
\hline 1910. & $173,400,000$ & $4,306,200,000$ & $58,000,000$ & $4,6,8,100,000$ \\
\hline 1913. & $199,100,000$ & $4,878,000,000$ & $68,500,000$ & $5,729,300,000$ \\
\hline 1920. & $201,200,000$ & $5,204,900,000$ & - & - \\
\hline 1923. & $936.800,000$ & $6,608,800,009$ & $64,800,000$ & $5,915,100,000$ \\
\hline 1995. & $292,700,000$ & $6,049,400,000$ & $70,700,000$ & $7,005,000,000$ \\
\hline
\end{tabular}

Les plus gros tonnages de marchandises transportés en 1925 se sont rangés comme suit : combustibles : 20,008,00o tonnes; produits métallurgiques : $7,560,000$ tonnes; produits de carrières : 5,538 , ooo tonnes; minerais : 
3,605,000 tonnes; produits de l'agriculture : 3,525,000 tonnes; chaux, plâtre et ciment : $2,537,000$ tonnes; sable : $2,204,000$ tonnes; bois divers : $1,928,000$ tonnes; engrais : I,717,000 tonnes; scories : 1,077,000 tonnes; briques : 934,000 tonnes; terres et terrils : 897,000 tonnes; produits alimentaires : 710,000 tonnes; bois de construction : 702,000 tonnes; produits céramiques : 562 ,ovo tonnes; textiles : 542,000 tonnes; verreries : $5 \mathrm{I} 2,000$ tonnes. Toutes les autres catégories de marchandises sont intervenues chacune pour moins de 500,000 tonnes.

Les chiffres, que nous avons reproduits à titre d'indication des coefficients d'exploitation, ont fait ressortir des résultats très inégaux dans les années qui se sont succédées depuis l'armistice et une grande différence entre ces résultats et ceux de l'avant-guerre. Les coefficients d'exploitation ne renseignent cependant que sur le rapport entre les dépenses et les recettes d'exploitation, abstraction faite de toutes charges financières. En tenant compte de celles-ci, on estimait avant la guerre que l'exploitation des chemins de fer de l'État belge se soldait sans grand bénéfice. Les réformes apportées dans l'établissement de la situation financière en 1905 ayant été appliquées de façon rétroactive aux résultats des exercices passés, on constata que, de 1835 jusqu'au 3 I décembre 1904 , l'exploitation des chemins de fer de l'État n'avait laissé qu'un solde actif de 25 I/2 millions. Ce fut une déception grave pour ceux qui, à l'exemple d'anciens ministres très écoutés, avaient comparé le chemin de fer à une "vache à lait ๖. La situation réelle était même encore moins favorable si l'on tenait compte des intérêts en compte courant avec le Trésor,du chef des sommes avancées au chemin de fer pendant les années de déficit. L'exposé des résultats de l'année I 906 concluait, après ces rectifications, à un excédent de déficits de près 
de 72 millions de francs. A la veille de la guerre, la situation ne s'était pas améliorée, puisque le bilan général au $3 \mathrm{I}$ décembre 1913 ramenait le solde actif de $25 \mathrm{I} / 2$ millions constatés en 1904 , à $\mathrm{I} 2 \mathrm{I} / 2$ millions. L'exercice $\mathrm{I} 9 \mathrm{r} 3$ avait, d'ailleurs, laissé un mali de $\mathrm{I}_{5} \mathrm{I} / 2$ millions. Le capital utile moyen en I913 ayant été de 2.728 millions, le revenu net des chemins de fer faisait ressortir le rendement du capital engagé à 3.15 p.c. C'était moins que la charge correspondant aux capitaux empruntés par l'État et engagés dans. le chemin de fer; de là le mali de I5 $\mathrm{I} / 2$ millions.

Depuis la guerre, les résultats de l'exploitation des chemins de fer de l'État belge ont fait l'objet d'une étude critique de la part du général $\mathrm{H}$. O. Mance et de M. Jules Jadot, spécialement commissionnés à cet effet par le Gouvernement. Cesrésultats ont été résumés dans les chiffres ci-après :

Recettes :

\begin{tabular}{|c|c|c|c|c|c|}
\hline 1 & & $\begin{array}{c}1913 . \\
-\end{array}$ & 1924. & 1925. & $\begin{array}{c}1926 . \\
\text { (estimatioe) }\end{array}$ \\
\hline & $=$ & 349 & 390 & 407 & 422 \\
\hline ipie & .1. & - & 1,626 & 1,695 & 1,859 \\
\hline
\end{tabular}

Dépenses d'après les comptes officiels :

$\begin{array}{rrrrr}\text { franes-or : * . . } & 246 & 348 & 379 & 389 \\ \text { francs-papier * . . . } & - & 4,450 & 1.578 & 1,619\end{array}$

Recettes nettes :

\begin{tabular}{|c|c|c|c|c|c|c|c|}
\hline franes-or. . & . . & $\cdot$ & . & 96 & 49 & 28 & 53 \\
\hline franes-papier & . . & . & . & - & 176 & 117 & 220 \\
\hline it: & 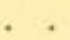 & , & " & 3.29 & 1.29 & 0.83 & 1.55 \\
\hline
\end{tabular}

MM. Mance et Jadot ont conclu pour l'année I924, à un déficit réel de 149 I/2 millions, en déduisant 87 r $/ 2$ millions de dépenses indiquées par les comptes officiels, mais en ajoutant 200 millions pour le fonds de renouvellement et 48 millions pour les fonds d'assurance et de réserve, et 
en assignant une somme de $\mathrm{r} 68 \mathrm{r} / 2$ millions au service de la dette.

Au point de vue particulier de la Société nationale des chemins de fer belges, la question se pose différemment. On sait, en effet, qu'aux termes de la loi qui a cédé l'exploitation à la nouvelle Société, l'État a conservé à sa charge la partie de la dette publique qui avait été consacrée à l'équipement des chemins de fer. Le rendement à envisager peut donc être basé sur la considération de la différence entre les recettes et les dépenses, en ajoutant à celles-ci les prélèvements nécessaires pour alimenter les fonds de renouvellement, d'assurance et de réserve. Tout l'excédent des recettes sur les dépenses revient par moitié à chacune des deux catégories d'actions privilégiées et d'actions ordinaires.

Les conditions du milieu économique dans lequel se présente l'exploitation des chemins de fer belges en eussent permis une exploitation fructueuse si la politique gouvernementale suivie en cette matière n'avait presque exclu la poursuite de bénéfices. De prime abord, la loi du I ${ }^{\text {er }}$ mai 1834 , créant le réseau des chemins de fer de l'État belge, disposa dans son article $5:$ " Les produits de la route provenant des péages, qui doivent être réglés annuellement par la loi, serviront à couvrir les intérêts et l'amortissement de l'emprunt ainsi que les dépenses annuelles d'entretien et d'administration s. La loi du $\mathrm{I}_{2}$ avril $\mathrm{I} 835$, concernant les péages et les réglements de police sur les chemins de fer (art. 4) avait confirmé ces dispositions : a Le produit des péages sera versé au trésor pour servir aux dépenses d'entretien et d'administration de la route, ainsi qu'au remboursement des intérêts et des capitaux affectés à sa construction. $)$

Il est d'ailleurs de bonne politique générale que l'État 
exploitant les chemins de fer, taxe les transports au prix de revient. L'État représente et ne doit connaître que l'intérêt public. Il ne peut distinguer entre les intérêts particuliers. C'est dire que s'il s'interdit, en intervenant en qualité d'exploitant dans le régime des transports, de lever des impôts d'une manière dissimulée, il ne doit ni gagner ni perdre à j'exploitation. S'il ne faut pas de bénéfice sur l'ensemble du rafic, il ne faut pas non plus, sur certaines catégories de transports, des bénéfices qui compenseraient des déficits sur d'autres.

Qu'il faille réprouver de la part de l'État la perception indirecte d'impôts sur les transports sous forme de tarifs bénéficiaires, c'est la conséquence même de son rôle dans la politique économique du pays. Pourrait-on admettre que l'État exerçant un monopole de transports, profitât de cette situation pour frapper les transports d'un impôt dont le résultat serait la réduction de l'ensemble du trafic, voire même la prohibition de certains trafics spéciaux? Ce serait l'opposé même du but qui doit être atteint par l'État, à savoir le développement maximum de la production et des échanges. Il méconnaitrait l'une des premières conditions de la prospérité publique.

Aussi, dans le rapport présenté au Parlement belge en rgc6, sur le Budget du Ministère des chemins de fer, au nom de la Section centrale, l'un des parlementaires les plus autorisés en la matière, s'exprimait en ces termes: \& Les chemins de fer ne sont pas créés pour être une source d'impôts indirects; c'est surtout un service public; qu'ils demandent la juste rémunération du service rendu, cela est non seulement équitable, mais il est impossible de faire des avantages aux voyageurs et aux transporteurs au détrimen du Trésor. Mais lorsqu'ils ont couvert leurs frais, l'intérè de l'amortissement du capital engagé, le surplus doit retour- 
ner en améliorations du service, réduction des tarifs et relèvement des traitements et salaires, en commençant par ceux qui sont les moins élevés. »

Ce principe a été consacré dans le régime appliqué aux chemins de fer fédéraux suisses. Aux termes du Règlement d'exécution du 7 novembre 1899 , leur produit net est affecté en premier lieu au paiement des intérêts et de l'amortissement de leur dette : le surplus est employé exclusivement dans l'intérêt des Chemins de fer fédéraux, à raison de 20 p. c. à verser dans un fonds de réserve jusqu'à concurrence de 50 millions de francs et de 80 p. c. pour améliorer les conditions de transport et pour réduire les tarifs.

Malgré des velléités de faire produire à ses chemins de fer un excédent au profit du Trésor et malgré les soldes bénéficiaires de certaines années d'exploitation, l'État belge s'est tenu d'assez près à la politique que nous venons d'esquisser. Aussi ses tarifs étaient-ils particulièrement bon marché avant la guerre. Il n'y avait aucun pays au monde où la tarification des transports par chemin de fer fut aussi modique que dans notre pays.

Ce bon marché des tarifs a subsisté après la guerre. En dépit des fluctuations dues aux variations dans la dépréciation du franc belge par rapport à l'or, ils sont restés notablement inférieurs en valeur-or à leur montant d'avantguerre. Cependant le I5 mars 1926, à l'époque de la rédaction de leur rapport déjà cité, MM. Mance et Jadot estimaient que les tarifs pour voyageurs représentaient en Belgique 80 à 85 p.c., exprimés en or, des tarifs d'avant la guerre, tandis qu'en Grande-Bretagne, par exemple, ils atteignaient 150 p.c. Quant aux marchandises, certains tarifs importants, par exenple, pour le charbon étaient, en or, notablement en dessous du niveau d'avant-guerre. Dans l'ensemble, les tarifs marchandises n'avaient augmenté 
que de 2 p.c. en moyenne, calculés en or, tandis que l'augmentation avait été de 50 à 55 p.c. en Grande-Bretagne et de 45 p.c. en Allemagne (en y comprenant l'impôt sur les transports). La forte dépréciation du franc belge eut bientôt pour conséquence de ramener tous les prix de transport évalués en or très au-dessous de leurs tarifs de I9I4.

La question a complètement changé par le fait de la constitution de la Société nationale des chemins de fer belges. Si pour les raisons exposées au sujet de l'Etat, celui-ci ne doit pas, en bonne politique, retirer des ressources de l'exploitation des chemins de fer, rien de plus normal qu'une compagnie exploitant un système de transports et fixant des tarifs de façon à obtenir un profit aussi élevé que possible. Telle sera d'ailleurs la faculté de la Société nationale des chemins de fer belges, sous réserve de la sauvegarde de l'intérêt public prévue dans la loi qui l'a créée. L'article 16 dispose, en effet, que les tarifs seront réglés par le Conseil d'administration, mais que le Gouvernement aura toujours le droit d'en exiger l'abaissement ou d'en interdire le relèvement. De plus, dans le cas de la Société nationale des chemins de fer belges, les chemins de fer constituent, à toute évidence, un instrument fiscal. N'est-ce pas, en effet, de leur rendement que dépendront la compensation pour l'Etat du paiement de l'intérêt qu'il a garanti aux porteurs d'actions privilégiées et l'octroi d'un superdividende à leur profit? Or, à l'attrait offert par ce superdividende sont étroitement liés le placement de ces mêmes titres et le versement au Fonds d'amortissement du produit de leur réalisation.

Dans leur rapport, MM. Mance et Jadot, en se basant sur le rendement net prévu dans le plan Dawes pour la Compagnie des chemins de fer allemands, estimaient que les che- 
mins de fer de l'Etat belge devaient pouvoir rendre annuellement 500 millions de francs-papier.

Une communication de la Société nationale des chemins de fer belges à la presse faisait valoir, en octobre 1926 , que, sur la base des résultats acquis pendant le premier semestre de l'année en cours, compte tenu des augmentations de tarifs de juin et d'août, le bénéfice probable de l'exercice 1926 serait d'environ 380 millions de francs. Après défalcation de 270 millions nécessaires pour versement aux fonds de renouvellement, d'assurances et de réserve, il resterait un solde distribuable de I ro millions. Si les tarifs de 1914 étaient appliqués en valeur-or aux transports effectués en 1926 , le bénéfice probable atteindrait 700 millions.

Le relèvement des tarifs n'a d'ailleurs pas tardé. En septembre 1926, le Ministre des Chemins de fer a ratifié, sur proposition du Conseil d'administration de la Société nationale, une majoration maximum de 25 p. c. des tarifs, en limitant les tarifs ainsi majorés au niveau d'avantguerre, et depuis le $\mathrm{I}^{\mathrm{er}}$ octobre tous les tarifs ont été majorés de 25 p.c., à l'exception du tarif I A, qui s'applique aux marchandises de grande valeur transportées par 5 tonnes, Pour celles-ci, l'augmentation n'a été que de 15 p. c., en raison de la crainte de concurrence des transports automobiles. Dans l'esprit du Conseil d'administration, cette augmentation exigée par les nécessités immédiates de rémunération des actions de la Société nationale des chemins de fer belges, a un caractère provisoire. Elle sera appliquée, en attendant qu'il soit possible de terminer la revision générale de la tarification en vigueur et d'apprécier les effets de la réorganisation des services.

La révision générale de la tarification pourra utilement s'inspirer du principe généralement suivi dans les exploi- 
tations de chemins de fer par des compagnies privées. Celles-ci ne peuvent réaliser de profits en taxant également tous les transports, car un même tarif appliqué aux diverses catégories de transports et de marchandises devrait être nécessairement très bas. S'il n'était pas très bas, en effet, beaucoup de produits seraient écartés du trafic, parce que leur transport ne laisserait plus de bénéfice à l'usager du chemin de fer par la différence de prix entre les points de départ et d'arrivée. D'un autre côté, un tarif uniformément bas ne procurerait à l'exploitant qu'un profit dérisoire.

Pour que le profit soit maximum - et il est normal qu'il en soit ainsi sous un régime d'exploitation privée - il faut que, d'un côté, les marchandises auxquelles le transport ajoute une grande plus-value soient taxées proportionnellement à celle-ci et que, de l'autre côté, celles desquelles le transport accroît peu la valeur, ne soient que faiblement taxées. Tel est lè principe qui consiste à faire payer à chaque marchandise ce qu'elle peut supporter.

Suivant ce principe les tarifs actuels des chemins de fer belges paraissent laisser encore, même après les augmentations du $1^{\text {er }}$ octobre 1926 , une grande marge pour de futures augmentations. Cette conclusion se dégage nettement de l'aperçu du rapport existant entre le prix du transport et la valeur de certains produits de grand trafic. 


\section{Nature des} marchandises.

\author{
Valeur \\ à la tonne.
}

1913 Oetobre
Prix du transport

à $80 \mathrm{~km}$.

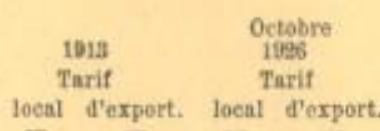

local d'export. local d'export.

\begin{tabular}{|c|c|c|c|c|c|c|c|c|c|c|}
\hline pot & & & & .20 & 2.70 & 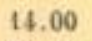 & & 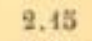 & 3.87 & 1.44 \\
\hline eurre. & 4000 & 25,000 & & 10 & 90 & 51.90 & 0 & 0.20 & 0.90 & 0.20 \\
\hline afé & 1550.00 & 16,400 & .00 & - & .90 & - & 0.58 & - & 31 & - \\
\hline ons dom & 2000 & 215 & 60 & 200 & .80 & 11.30 & 1800 & 0.00 & .20 & 5.25 \\
\hline 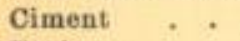 & 27.00 & 170 & & 1.95 & 0 & 18.80 & 3 & 7.92 & 49.23 & 1105 \\
\hline arine. . & 390.00 & 3,000 & 5.65 & - & 0 & - & 1.76 & - & .09 & - \\
\hline taine brute . & $4600 \quad 00$ & 24,000 & 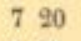 & - & 0 & - & .45 & - & .21 & - \\
\hline Haìs. . . & 15650 & 1, & 5 & - & 32.70 & - & 3.61 & - & .63 & - \\
\hline finerais de fer & 4.75 & 31.70 & 1.85 & - & 8.40 & - & 38.94 & - & 96.30 & - \\
\hline e... & . 290.00 & 2,580 & 9.00 & - & 1.90 & - & 4.09 & - & 2.01 & + \\
\hline Pommes de terr & 6370 & 550 & 4.88 & 410 & 32.70 & 32.70 & 7.51 & 6.43 & 5.94 & 594 \\
\hline . . . & 149.50 & 950 & 5.65 & 3.20 & 32.70 & 14.00 & 3.77 & 9.14 & 3.44 & 1.47 \\
\hline cories Thomas & 41.70 & 940 & 2.60 & 2.00 & 17.00 & 23.70 & 6.24 & 4.79 & 7.08 & 9.87 \\
\hline ere cristallis & 340.00 & 850 & 9.00 & 8. 10 & 51.90 & 51,90 & 9.63 & 2.39 & 1.82 & 1.89 \\
\hline
\end{tabular}

Ces chiffres font voir à suffisance que pour nombre de marchandises donnant lieu à un gros volume de transports le rapport du prix du transport à la valeur de la marchandise est encore très inférieur à sa valeur d'avant-guerre. C'est dire que les tarifs pourraient éventuellement être majorés sans peser indûment sur le trafic

L'exposé objectif auquel nous venons de nous livrer ne laisse aucun doute sur les possibilités de rémunération du capital engagé dans l'exploitation du réseau de la Société nationale des chemins de fer belges.

Des bénéfices seront assurément réalisables dans une proportion qui suffira à une large rémunération de ce capital, par la hausse ou le remaniement des tarifs. Ce n'est pas à dire que la hausse des tarifs soit la condition indispensable à la rémunération du capital. Des réductions de tarifs seront même sans doute possibles sans compro- 
mettre en rien les résultats financiers attendus de la constitution de la Société nationale des chemins de fer belges. Celle-ci a été instituée à l'effet de réaliser des progrès dans l'exploitation des chemins de fer par une large indépendance vis-à-vis de l'Etat et du Parlement. L'exploitation pourra se poursuivre dans un esprit de saine gestion industrielle à labri des intrusions politiques et des i fluences électorales. Un comité permanent a été institué en vertu des statuts pour pourvoir à la gestion journalière mieux qu'un conseil d'administration réunissant un grand nombre de membres en des séances longuement espacées.

Dans ces dernières années l'ancienne administration des chemins de fer l'Etat avait réalisé de grand progrès dans le sens de l'économie de l'exploitation. On peut les caractériser par quelques chiffres :

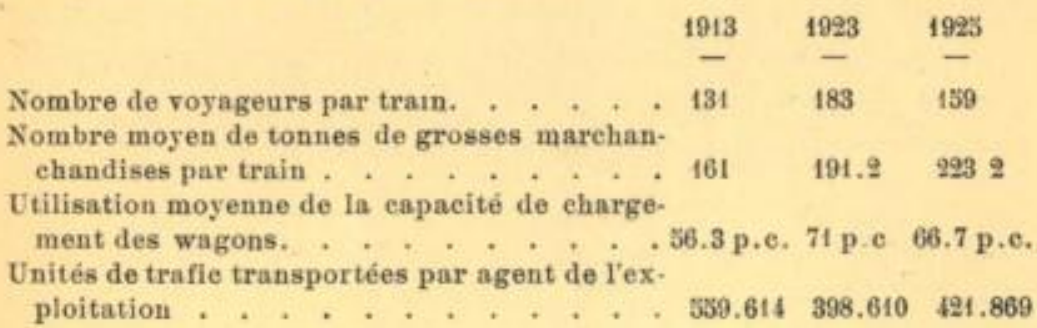

Le nombre moyen de voyageurs par train est l'indication d'une accomodation plus ou moins stricte du service des trains au nombre de personnes à transporter. A cet égard le chiffre de 1925 montre une meilleure utilisation que celle constatée en 1913; par contre, l'utilisation était meilleure en 1923 qu'en 1925. La diminution constatée en rg25 est la conséquence, au dire de l'Administration, de mesures qui, tout en tenant compte du principe d'économie devant régir l'exploitation, ont eu pour but, dans l'intérêt général, de créer des communications rapides et adaptées le mitux possible aux besoins de la population. 
Le nombre moyen de tonnes de grosses marchandises par train montre un grand accroissement de rgr 3 a 1925. On doit y voir surtout l'indice d'une meilleure utilisation du personnel attribué à chaque train.

L'utilisation moyenne de la capacité de chargement des wagons a été de 10 p. c. supérieure en 1925 par rapport à 1913; c'est dire que le matériel est mieux utilisé; le chiffre de 1925 est cependant inférieur à celui de 1923 .

Au point de vue du rendement du personnel il est intéressant de mettre en rapport le nombre de voyageurs et de tonnes de marchandises transportés sur toute leur distance et le nombre d'agents de l'exploitation. Ce rapport est exprimé par le chiffre des unités de trafic transportées par agent, en entendant par unité de trafic, le transport d'un voyageur ou d'une tonne de marchandises sur un kilomètre avec réduction d'un voyageur à 0.8 tonne de grosses marchandises. Il avait fortement diminué en 1923 en comparai son de rgr3.La diminution a été due principalement à l'instauration du régime des huit heures de travail; mais les améliorations apportées à l'utilisation du personnel ont permis d'obtenir en 1925 un chiffre sensiblement supérieur à celui de 1923 .

On pourrait ajouter d'autres économies dues à la meilleure organisation du travail des ateliers et se traduisant, par exemple, par une diminution sensible du temps de travail d'atelier subi en moyenne par une locomotive ou un 'wagon renvoyé à l'atelier de reparation. D'autres progrès sont marqués par la plus longue durée d'utilisation d'une locomotive entre deux passages consécutifs à l'atelier de réparations, etc.

La nouvelle Société nationale des chemins de fer belges est en mesure de hâter ou de généraliser ces progrès. Ce fut d'ailleurs l'un des objectifs essentiels de sa création, 
puisque celle-cià visé à réaliser l'autonomie industrielle de l'exploitation des chemins de fer autant qu'à poursuivre des fins financières propres Quel que fut l'esprit d'organisation des chefs de l'ancienne administration, ils étaient entravés dans leur action par les influences gouvernementales e parlementaires. Sous l'impulsion d'un conseil d'administration indépendant et d'un comité permanent vigilant, ils pourront réaliser de nouveaux et rapides progrès. Ceux-ci contribueront à consacrer dans l'opinion publique la supériorité de la forme d'exploitation des chemins de fer de l'Etat qui a été innovée en Belgique. Ils coopéreront, dans l'intérêt des finances publiques, au rendement que l'on est en droit d'attendre du réseau des chemins de fer belges en considération de la richesse du milieu économique, unique au monde, constitué par la Belgique et ses régions limitrophes.

Georges De Leener, Professeur a l'Universite de Bruxelles. 